\title{
Olanzapine for the Prevention of Chemotherapy-Induced Nausea and Vomiting
}

\author{
Rudolph M. Navari, M.D., Rui Qin, Ph.D., Kathryn J. Ruddy, M.D., Heshan Liu, Ph.D., Steven \\ F. Powell, M.D., Madhuri Bajaj, M.D., Leah Dietrich, M.D., David Biggs, M.D., Jacqueline M. \\ Lafky, M.S., and Charles L. Loprinzi, M.D. \\ Indiana University School of Medicine-South Bend, South Bend (R.M.N.); Alliance Statistics and \\ Data Center, Mayo Clinic (R.Q., H.L.), and Mayo Clinic (K.J.R., J.M.L., C.L.L.), Rochester, MN; \\ Sanford NCORP (National Cancer Institute Community Oncology Research Program) of the \\ North Central Plains, Sioux Falls, SD (S.F.P.); Illinois Cancer Care-Peoria, Peoria (M.B.); \\ Gundersen Lutheran Medical Center, La Crosse, WI (L.D.); and Delaware-Christiana Care \\ NCORP, Newark, DE (D.B.)
}

\section{Abstract}

BACKGROUND-We examined the efficacy of olanzapine for the prevention of nausea and vomiting in patients receiving highly emetogenic chemotherapy.

\begin{abstract}
METHODS-In a randomized, double-blind, phase 3 trial, we compared olanzapine with placebo, in combination with dexamethasone, aprepitant or fosaprepitant, and a 5-hydroxytryptamine type 3-receptor antagonist, in patients with no previous chemotherapy who were receiving cisplatin ( $\geq 70 \mathrm{mg}$ per square meter of body-surface area) or cyclophosphamide-doxorubicin. The doses of the three concomitant drugs administered before and after chemotherapy were similar in the two groups. The two groups received either $10 \mathrm{mg}$ of olanzapine orally or matching placebo daily on days 1 through 4 . Nausea prevention was the primary end point; a complete response (no emesis and no use of rescue medication) was a secondary end point.
\end{abstract}

RESULTS-In the analysis, we included 380 patients who could be evaluated (192 assigned to olanzapine, and 188 to placebo). The proportion of patients with no chemotherapy-induced nausea was significantly greater with olanzapine than with placebo in the first 24 hours after chemotherapy ( $74 \%$ vs. $45 \%, \mathrm{P}=0.002)$, the period from 25 to 120 hours after chemotherapy ( $42 \%$ vs. $25 \%, \mathrm{P}=0.002)$, and the overall 120 -hour period ( $37 \%$ vs. $22 \%, \mathrm{P}=0.002$ ). The complete-response rate was also significantly increased with olanzapine during the three periods: $86 \%$ versus $65 \%(\mathrm{P}<0.001), 67 \%$ versus $52 \%(\mathrm{P}=0.007)$, and $64 \%$ versus $41 \%(\mathrm{P}<0.001)$, respectively. Although there were no grade 5 toxic effects, some patients receiving olanzapine had increased sedation (severe in 5\%) on day 2.

CONCLUSIONS-Olanzapine, as compared with placebo, significantly improved nausea prevention, as well as the complete-response rate, among previously untreated patients who were

\footnotetext{
Address reprint requests to Dr. Navari at 4518 Crown Point Ln., Mount Olive, AL 35117, or at rmnavari@gmail.com. Disclosure forms provided by the authors are available with the full text of this article at NEJM.org.
} 
receiving highly emetogenic chemotherapy. (Funded by the National Cancer Institute; ClinicalTrials.gov number, NCT02116530.)

Chemotherapy-induced nausea and vomiting are associated with a significant deterioration in quality of life and are perceived by patients as major adverse effects of cancer treatment. ${ }^{1}$ The use of 5-hydroxytryptamine type $3\left(5-\mathrm{HT}_{3}\right)$ receptor antagonists, ${ }^{2}$ dexamethasone, ${ }^{2}$ and neurokinin-1 $\left(\mathrm{NK}_{1}\right)$ receptor antagonists ${ }^{3-9}$ has significantly improved the control of this troublesome side effect. International guidelines ${ }^{10-12}$ recommend combinations of these agents to prevent chemotherapy-induced nausea and vomiting in patients receiving moderately or highly emetogenic chemotherapy. Nonetheless, nausea remains a major problem for many patients. ${ }^{1,2}$

Olanzapine is approved by the Food and Drug Administration (FDA) as an antipsychotic agent that blocks multiple neurotransmitters: dopamine at $\mathrm{D}_{1}, \mathrm{D}_{2}, \mathrm{D}_{3}$, and $\mathrm{D}_{4}$ receptors; serotonin at 5-HT type 2a, 5-HT type 2c (5- $\left.\mathrm{HT}_{2 \mathrm{c}}\right), 5-\mathrm{HT}_{3}$, and 5-HT type 6 receptors; catecholamines at alpha $a_{1}$-adrenergic receptors; acetylcholine at muscarinic receptors; and histamine at $\mathrm{H}_{1}$ receptors in the central nervous system. ${ }^{8,9,13}$ Side effects may include mild short-term sedation, ${ }^{13-15}$ as well as weight gain and an increased risk of diabetes mellitus with prolonged use ( $>6$ months).${ }^{15-17}$ The activity of olanzapine at multiple receptors, particularly the $\mathrm{D}_{2}, 5-\mathrm{HT}_{2 \mathrm{c}}$, and $5-\mathrm{HT}_{3}$ receptors, which may be involved in nausea and vomiting, suggests that it might have clinically significant antiemetic properties.

A single-institution phase 3 trial showed that olanzapine, when combined with a single dose of dexamethasone and a single dose of palonosetron, an $\mathrm{HT}_{3}$-receptor blocker, was effective in controlling early and longer-term nausea and vomiting in patients receiving highly emetogenic chemotherapy. ${ }^{18}$ Additional single-institution phase 3 studies have shown that olanzapine combined with standard antiemetic agents improves control of nausea and vomiting in patients receiving moderately or highly emetogenic chemotherapy. ${ }^{19,20}$ A recent review, however, pointed to possible methodologic issues in these single-institution studies and called for well-planned, randomized, double-blind, multicenter studies to evaluate the role of olanzapine in the prevention of chemotherapy-induced nausea and vomiting. ${ }^{21}$

The primary objective of the current trial was to evaluate olanzapine, as compared with placebo, for the control of nausea in patients receiving highly emetogenic chemotherapy, with nausea prevention assessed during three periods: 0 to 24 hours, 25 to 120 hours, and 0 to 120 hours after chemotherapy. Secondary objectives were to compare the two study groups for the number of patients with a complete response (no emesis and no rescue therapy) in the three periods, as well as to evaluate potential toxic effects of olanzapine.

\section{Methods}

\section{Eligibility Criteria}

Patients 18 years of age or older with malignant disease who had not received previous chemotherapy were eligible for enrollment in the study if they were scheduled to receive highly emetogenic chemotherapy (either cisplatin at a dose $\geq 70 \mathrm{mg}$ per square meter of body-surface area, with or without other chemotherapeutic agents, or doxorubicin at a dose 
of $60 \mathrm{mg}$ per square meter plus cyclophosphamide at a dose of $600 \mathrm{mg}$ per square meter) and had a European Cooperative Oncology Group (ECOG) performance status of 0, 1, or 2 (on a 5-point scale, with 0 indicating no symptoms and higher numbers indicating increasing disability). Additional eligibility criteria were a serum creatinine level of $2.0 \mathrm{mg}$ per deciliter (177 $\mu \mathrm{mol}$ per liter) or less, an aspartate or alanine aminotransferase level that was no more than 3 times the upper limit of the normal range, and an absolute neutrophil count of at least 1500 per cubic millimeter; no nausea or vomiting in the 24 hours before enrollment; no severe cognitive compromise; no known history of central nervous system disease (e.g., brain metastases or a seizure disorder); no treatment with another antipsychotic agent such as risperidone, quetiapine, clozapine, a phenothiazine, or a butyrophenone within 30 days before enrollment or plans for such treatment during the study period; no long-term use of a phenothiazine as an antipsychotic agent (patients could receive prochlorperazine and other phenothiazines as rescue antiemetic therapy); no concurrent use of amifostine; no concurrent abdominal radiotherapy; no concurrent use of quinolone antibiotic therapy; no chronic alcoholism; no known hypersensitivity to olanzapine; no known cardiac arrhythmia, uncontrolled congestive heart failure, or acute myocardial infarction within the previous 6 months; and no history of uncontrolled diabetes mellitus.

Because of the teratogenic potential of the therapy used in this trial, women of childbearing age had to have a negative result of a pregnancy test performed within 7 days before enrollment. They also had to agree to use appropriate birth control throughout their participation in the study.

\section{Study Design and Oversight}

Patients were assigned to a study group with the use of the Pocock and Simon dynamic randomization procedure, which balances the marginal distributions of the stratification factors between study groups. The stratification factors were sex, chemotherapy regimen (cisplatin-containing regimen vs. anthracycline plus cyclophosphamide), and the specific 5$\mathrm{HT}_{3}$-receptor antagonist used (palonosetron, ondansetron, or granisetron). The patients and the medical professionals who cared for them were unaware of the assigned study regimen. The patients were assessed for only one chemotherapy cycle. Rescue therapy of the treating investigator's choice was permitted for nausea, emesis, or retching, depending on the clinical circumstances.

All patients gave written informed consent. The study was approved by the institutional review board at each participating site and was independently monitored by the Alliance Data and Safety Monitoring Board. Data collection and analyses were conducted by the Alliance Statistics and Data Center. Data quality was ensured by a review of data performed by the Alliance Statistics and Data Center and by the study chairperson (the first author) according to Alliance policies. The authors vouch for the accuracy and completeness of the data and analysis and for adherence to the study protocol, available with the full text of this article at NEJM.org. 


\section{Treatment Regimen}

All participants received a $5-\mathrm{HT}_{3}$-receptor antagonist (palonosetron intravenously at a dose of $0.25 \mathrm{mg}$, granisetron intravenously at a dose of $1 \mathrm{mg}$ or orally at a dose of $2 \mathrm{mg}$, or ondansetron intravenously or orally at a dose of $8 \mathrm{mg}$, with the specific agent chosen by the primary clinician) on day 1 of chemotherapy, dexamethasone (12 mg orally on day 1 , and 8 mg orally on days 2,3 , and 4), and an $\mathrm{NK}_{1}$-receptor antagonist on day 1 . In addition, patients received olanzapine (10 mg per day orally) or a matching placebo on days 1 through 4. The $\mathrm{NK}_{1}$-receptor antagonist was intravenous fosaprepitant (150 mg on day 1) or oral aprepitant (125 mg on day 1 , and $80 \mathrm{mg}$ on days 2 and 3). The doses of the $5-\mathrm{HT}_{3}$-receptor antagonists, dexamethasone, and aprepitant used in the study are the standard doses recommended by various international guidelines for antiemetic agents. ${ }^{10-12}$ The dose of olanzapine was chosen on the basis of previous studies. ${ }^{2,18,19,22}$ In a phase 1 trial of olanzapine as a prophylactic antiemetic agent, Passik et al. ${ }^{22}$ determined that $10 \mathrm{mg}$ per day for 4 days was a dose associated with no toxic effects except minimal sedation. In a phase 2 trial, Navari et al. ${ }^{23}$ used a loading dose of olanzapine (administered daily for 2 days before chemotherapy) but subsequently determined that a loading dose was not necessary. ${ }^{18}$

\section{Study Visits and Assessment Procedures}

In the prestudy period, all pertinent demographic characteristics and medical data were recorded. Patients were asked to complete daily records of episodes of vomiting or retching (number and time) and the use of rescue therapy from the first day of chemotherapy (day 1) through day 5. Patients were also asked to record daily levels of nausea according to a visual-analogue scale ${ }^{24}$ ranging from 0 ("no nausea at all") to 10 ("nausea as bad as it can be"). A study nurse contacted each patient daily on days 2 through 5 to ask about toxic effects and remind the patient to complete forms. Adverse events were graded according to the terminology and grading categories defined in the National Cancer Institute's Common Terminology Criteria for Adverse Events, version 4.0. In addition, every day for 5 days, patients rated undesired sedation and undesired increase in appetite on numerical scales (with scores ranging from 0 to 10 , with higher scores indicating greater sedation or appetite increase).

\section{Outcomes}

The primary end point, no nausea, was defined as a response of 0 on the visual-analogue scale for nausea during the overall assessment period (0 to 120 hours), the early assessment period ( 0 to 24 hours), and the later assessment period ( 25 to 120 hours). The proportion of patients with no nausea was compared between treatment groups sequentially: first for the overall period and then for the early and later periods concurrently. This hierarchical order was chosen because we considered relief for the overall period to be most important and relief in the early and later periods to be of equal secondary importance.

Secondary end points included a complete response (no emetic episodes and no use of rescue medication). A complete response was determined on the basis of the patients' daily records during the overall, early, and later assessment periods and reported adverse events. 


\section{Statistical Analysis}

A group sequential design with an interim analysis for superiority and futility was adopted for this trial. The interim analysis was to be conducted when $50 \%$ of patients had been enrolled and had completed their daily assessments of nausea and vomiting. We used the Lan-DeMets family ${ }^{25}$ of alpha- and beta-spending functions corresponding to the O'BrienFleming boundary to control for overall type I and type II error rates. At the interim analysis, a P value greater than 0.003 and less than 0.844 indicated that the trial would continue.

For the primary end point, we used chi-square tests to compare the proportion of patients with no nausea between treatment groups sequentially, first for the overall period, and then for the early and later periods together. A serial Simes gatekeeping procedure ${ }^{25}$ for the interim and final analyses was used to maintain the overall significance level at the level specified by the Lan-DeMets alpha-spending function. Logistic models were used to incorporate stratification factors, baseline scores, and other characteristics of the patients. The primary analysis did not include missing data because of the small number of patients with missing data for the primary end point, but a sensitivity analysis was performed to evaluate the robustness of the primary analysis.

The modified intention-to-treat principle ${ }^{26}$ was applied for the primary analysis of efficacy in the population of patients who could be evaluated. This population was defined as all patients meeting the eligibility criteria who did not withdraw from the study before receiving treatment and had no major protocol violations (see the Supplementary Appendix, available at NEJM.org).

A previous study ${ }^{18}$ showed that the proportion of patients not receiving olanzapine who were free of nausea during the overall assessment period was about $40 \%$. Considering that a $17.5 \%$ increase in this proportion would be a clinically meaningful effect size, we calculated that we would need to enroll 332 patients (166 per group) to achieve $90 \%$ power to detect this effect size at the 5\% significance level, using a two-sided chi-square test for a fixed sample size. We increased the sample size to 338 patients (169 per group) after adding an interim analysis for superiority and futility. The sample size calculation and simulation for operating characteristics of the group sequential design were conducted with the use of East software, version 5.4 (Cytel).

Analyses of secondary end points were exploratory in nature, and reported $\mathrm{P}$ values for these analyses have therefore not been adjusted for multiple comparisons. Growth-curve models were used for repeated measures of symptoms and secondary end points.

\section{Results}

\section{Study Patients}

Figure 1 (and Fig. S1 in the Supplementary Appendix) shows the distribution and randomization of the study patients. A total of 401 patients at 46 academic or community practice institutions in the United States were randomly assigned to a study group between August 2014 and March 2015; 380 patients (192 assigned to olanzapine and 188 assigned to placebo) began the study. 
Demographic and clinical characteristics are presented in Table 1 for the 380 patients who began the study and who did not have major protocol violations. There were no significant differences between the olanzapine and placebo groups in terms of age, race, sex, 5- $\mathrm{HT}_{3}-$ receptor inhibitor received, chemotherapy regimen administered, ECOG performance status, or primary site of disease. In each group, a majority of the patients were women and a majority received chemotherapy consisting of doxorubicin plus cyclophosphamide. The distribution of institutions was balanced between the study groups.

\section{Efficacy}

An interim analysis was performed in February 2015, after primary end-point data had been recorded for $50 \%$ of the patients on the basis of their daily assessments of nausea and vomiting. A $P$ value of 0.3599 for the chi-square test comparing the two study groups with respect to the proportion of patients who had no nausea during the overall assessment period indicated that we should continue enrolling patients according to the group sequential design. At the final analysis, the proportion of patients who had no nausea (the primary end point) was significantly greater in the olanzapine group than in the placebo group in each of the three assessment periods: early period, $74 \%$ vs. $45 \%(\mathrm{P}=0.002)$; later period, $42 \%$ vs. $25 \%(\mathrm{P}=0.002)$; and overall period, $37 \%$ vs. $22 \%(\mathrm{P}=0.002)$ (Table 2$)$. When all the patients with missing data were considered to have had nausea, the results were similar, with $P$ values of less than 0.004 for the early, later, and overall periods.

Likewise, there were significant differences between the olanzapine and placebo groups with respect to freedom from clinically significant nausea - that is, a nausea score of less than 3 on a scale from 0 to 10 , as defined in previous reports. ${ }^{1,2,6,27-29}$ The proportion of patients receiving olanzapine who had no clinically significant nausea was $87 \%$, versus $70 \%$ of those receiving placebo, in the early period $(\mathrm{P}=0.001), 72 \%$ versus $55 \%$ in the later period $(\mathrm{P}=$ $0.001)$, and $67 \%$ versus $49 \%$ in the overall period $(\mathrm{P}=0.001)$.

The proportion of patients with a complete response was significantly higher in the olanzapine group than in the placebo group in all three assessment periods. During the early, later, and overall periods, the proportions were $86 \%$ vs. $65 \%$ ( $\mathrm{P}<0.001), 67 \%$ vs. $52 \%$ ( $\mathrm{P}=$ $0.007)$, and $64 \%$ vs. $41 \%(\mathrm{P}<0.001)$, respectively (Table 3$)$.

\section{Adverse Events}

There were two grade 3 adverse events (fatigue and hyperglycemia) in the olanzapine group and two grade 3 adverse events (abdominal pain and diarrhea) in the placebo group. There were three grade 4 adverse events (two of which were hematologic) in the olanzapine group and no grade 4 adverse events in the placebo group. No grade 5 adverse events were reported in the study. None of the grade 3 or 4 adverse events were attributed to olanzapine by the attending clinician.

Figure 2A shows that patients receiving olanzapine, as compared with those receiving placebo, had significantly increased sedation (severe in 5\%) on day 2 as compared with baseline. The sedation resolved on days 3,4 , and 5 , even though patients continued to receive olanzapine on days 3 and 4 . No patient discontinued the study because of undesired sedation. Figure $2 \mathrm{~B}$ shows that there were no significant differences between the olanzapine 
and placebo groups with respect to undesired increase in appetite on days 2 through 5 after chemotherapy as compared with baseline.

\section{Discussion}

This large, randomized, double-blind, placebo-controlled, phase 3 trial showed that it is more effective to combine olanzapine than placebo with an $\mathrm{NK}_{1}$-receptor antagonist, a 5$\mathrm{HT}_{3}$-receptor antagonist, and dexamethasone for the prevention of nausea and vomiting in patients who have not received previous chemotherapy but are currently receiving highly emetogenic chemotherapy. Patients who received olanzapine were more likely than those who received placebo to be free of nausea and emesis in the early, later, and overall assessment periods.

The benefit of olanzapine in controlling nausea and emesis has been suggested in other phase 3 trials. ${ }^{19,20}$ These trials showed that when olanzapine was added to guidelinedirected prophylactic agents, nausea and emesis were significantly reduced. The efficacy of olanzapine for nausea control contrasts with the findings in clinical trials of $\mathrm{NK}_{1}$-receptor antagonists. Although these agents (aprepitant, fosaprepitant, netupitant, and rolapitant) significantly controlled early and later emesis in patients receiving moderately or highly emetogenic chemotherapy they appear to have been less effective in controlling nausea. ${ }^{1-7,18}$

In our study, patients who received olanzapine had more drowsiness on day 2 than at baseline, but for the most part, this symptom abated on days 3, 4, and 5, despite continued administration of oral olanzapine on days 3 and 4 , suggesting that the patients adapted to the sedative effect of olanzapine. Undesired increase in appetite was not seen in the current trial. There were no serious adverse events related to olanzapine, and no patient discontinued olanzapine because of toxic effects. In view of the temporary drowsiness reported in this trial and previous reports of temporary drowsiness, ${ }^{13-15}$ more detailed information on drowsiness ratings, as well as the use of a lower dose of olanzapine $(5 \mathrm{mg})$, could be explored in future trials.

A limitation of our study is that we evaluated only one dose level of olanzapine. Lower or higher doses may have an effect on efficacy, toxic effects, or both. In addition, the study did not address the efficacy of olanzapine for multiple chemotherapy cycles. These issues should be considered in future clinical trials. In conclusion, our study showed that olanzapine combined with an $\mathrm{NK}_{1}$-receptor antagonist, a 5- $\mathrm{HT}_{3}$-receptor antagonist, and dexamethasone is more effective than placebo combined with these agents for the prevention of nausea and vomiting in patients with no previous chemotherapy who are receiving highly emetogenic chemotherapy.

\section{Supplementary Material}

Refer to Web version on PubMed Central for supplementary material.

\section{Acknowledgments}

The content of this article is solely the responsibility of the authors and does not necessarily represent the official views of the National Institutes of Health. 
Supported by grants from the National Cancer Institute (UG1CA189823, U10CA180790, UG1CA189830, UG1CA189825, UG1CA189956, CA035269, and 5UG1CA189819).

\section{References}

1. Ng TL, Hutton B, Clemons M. Chemotherapy-induced nausea and vomiting: time for more emphasis on nausea? Oncologist. 2015; 20:576-583. [PubMed: 25948677]

2. Navari RM. Management of chemotherapy-induced nausea and vomiting: focus on newer agents and new uses for older agents. Drugs. 2013; 73:249-262. [PubMed: 23404093]

3. Aapro M, Carides A, Rapoport BL, Schmoll HJ, Zhang L, Warr D. Aprepitant and fosaprepitant: a 10-year review of efficacy and safety. Oncologist. 2015; 20:450-458. [PubMed: 25795636]

4. Hesketh PJ, Rossi G, Rizzi G, et al. Efficacy and safety of NEPA, an oral combination of netupitant and palonosetron, for prevention of chemotherapy-induced nausea and vomiting following highly emetogenic chemotherapy: a randomized dose-ranging pivotal study. Ann Oncol. 2014; 25:13401346. [PubMed: 24608196]

5. Aapro M, Rugo H, Rossi G, et al. A randomized phase III study evaluating the efficacy and safety of NEPA, a fixed-dose combination of netupitant and palonosetron, for prevention of chemotherapyinduced nausea and vomiting following moderately emetogenic chemotherapy. Ann Oncol. 2014; 25:1328-1333. [PubMed: 24603643]

6. Navari RM. Profile of netupitant/palonosetron (NEPA) fixed dose combination and its potential in the treatment of chemotherapy-induced nausea and vomiting (CINV). Drug Des Devel Ther. 2015; 9:155-161.

7. Navari RM. Rolapitant for the treatment of chemotherapy-induced nausea and vomiting. Expert Rev Anticancer Ther. 2015; 15:1127-1133. [PubMed: 26366937]

8. Navari RM. Olanzapine for the prevention and treatment of chronic nausea and chemotherapyinduced nausea and vomiting. Eur J Pharmacol. 2014; 722:180-186. [PubMed: 24157985]

9. Hocking CM, Kichenadasse G. Olanzapine for chemotherapy-induced nausea and vomiting: a systematic review. Support Care Cancer. 2014; 22:1143-1151. [PubMed: 24522741]

10. Basch E, Prestrud AA, Hesketh PJ, et al. Antiemetics: American Society of Clinical Oncology clinical practice guideline update. J Clin Oncol. 2011; 29:4189-4198. [PubMed: 21947834]

11. Clinical practice guidelines in oncology, version 1: anti-emesis. Fort Washington, PA: National Comprehensive Cancer Network; 2015. (https://www.nccn.org/professionals/physician_gls/ f_guidelines.asp)

12. MASCC/ESMO antiemetic guidelines. Hillerod, Denmark: Multinational Association of Supportive Care in Cancer; 2013. (http://www.mascc.org/antiemetic-guide-lines)

13. Bymaster FP, Calligaro DO, Falcone JF, et al. Radioreceptor binding profile of the atypical antipsychotic olanzapine. Neuropsychopharmacology. 1996; 14:87-96. [PubMed: 8822531]

14. Bymaster FP, Falcone JF, Bauzon D, et al. Potent antagonism of 5-HT3 and 5-HT6 receptors by olanzapine. Eur J Pharmacol. 2001; 430:341-349. [PubMed: 11711053]

15. Allison DB, Casey DE. Antipsychotic-induced weight gain: a review of the literature. J Clin Psychiatry. 2001; 62(Suppl 7):22-31.

16. Hale AS. Olanzapine. Br J Hosp Med. 1997; 58:442-445. [PubMed: 9619205]

17. Goldstein LE, Sporn J, Brown S, et al. New-onset diabetes mellitus and diabetic ketoacidosis associated with olanzapine treatment. Psychosomatics. 1999; 40:438-443. [PubMed: 10479950]

18. Navari RM, Gray SE, Kerr AC. Olanzapine versus aprepitant for the prevention of chemotherapyinduced nausea and vomiting: a randomized phase III trial. J Support Oncol. 2011; 9:188-195. [PubMed: 22024310]

19. Tan L, Liu J, Liu X, et al. Clinical research of olanzapine for prevention of chemotherapy-induced nausea and vomiting. J Exp Clin Cancer Res. 2009; 28:131. [PubMed: 19775450]

20. Mizukami N, Yamauchi M, Koike K, et al. Olanzapine for the prevention of chemotherapy-induced nausea and vomiting in patients receiving highly or moderately emetogenic chemotherapy: a randomized, double-blind, placebo-controlled study. J Pain Symptom Manage. 2014; 47:542-550. [PubMed: 23856100] 
21. Fonte C, Fatigoni S, Roila F. A review of olanzapine as an antiemetic in chemotherapy-induced nausea and vomiting and in palliative care patients. Crit Rev Oncol Hematol. 2015; 95:214-221. [PubMed: 25779971]

22. Passik S, Navari RM, Jung SH, et al. A phase I trial of olanzapine (Zyprexa) for the prevention of delayed emesis in cancer patients: a Hoosier Oncology Group study. Cancer Invest. 2004; 22:383388. [PubMed: 15493359]

23. Navari RM, Einhorn LH, Passik SD, et al. A phase II trial of olanzapine for the prevention of chemotherapy-induced nausea and vomiting: a Hoosier Oncology Group study. Support Care Cancer. 2005; 13:529-534. [PubMed: 15700131]

24. Börjeson S, Hursti TJ, Peterson C, et al. Similarities and differences in assessing nausea on a verbal category scale and a visual analogue scale. Cancer Nurs. 1997; 20:260-266. [PubMed: 9265812]

25. Jennison, C., Turnbull, BW. Group sequential methods with applications to clinical trials. Boca Raton, FL: Chapman and Hall/CRC Press; 1999.

26. Abraha I, Montedori A. Modified intention to treat reporting in randomised controlled trials: systematic review. BMJ. 2010; 340:c2697. [PubMed: 20547685]

27. Schwartzberg L, Aapro M, Hesketh PJ, et al. Do NK-1 receptor antagonists contribute to nausea control? Evaluation of the novel NEPA fixed-dose combination of NK-1 receptor antagonist plus a 5-HT3 receptor antagonist from pivotal trials. Support Care Cancer. 2014; 22(Suppl):S107.

28. Schwartzberg L, Modiana M, Rapoport B, et al. Safety and efficacy assessment of rolapitant for the prevention of chemotherapy-induced nausea and vomiting following administration of moderately emetogenic chemotherapy or anthracycline and cyclophosphamide regimens in patients with cancer: a randomised, active-controlled, double-blind, phase 3 trial. Lancet Oncol. 2015; 16:10711078. [PubMed: 26272768]

29. Rapoport B, Chasen M, Gridelli C, et al. Safety and efficacy assessment of rolapitant for the prevention of chemotherapy-induced nausea and vomiting after administration of cisplatin-based highly emetogenic chemotherapy in patients with cancer: two randomised, active-controlled, double-blind, phase 3 trials. Lancet Oncol. 2015; 16:1079-1089. [PubMed: 26272769] 


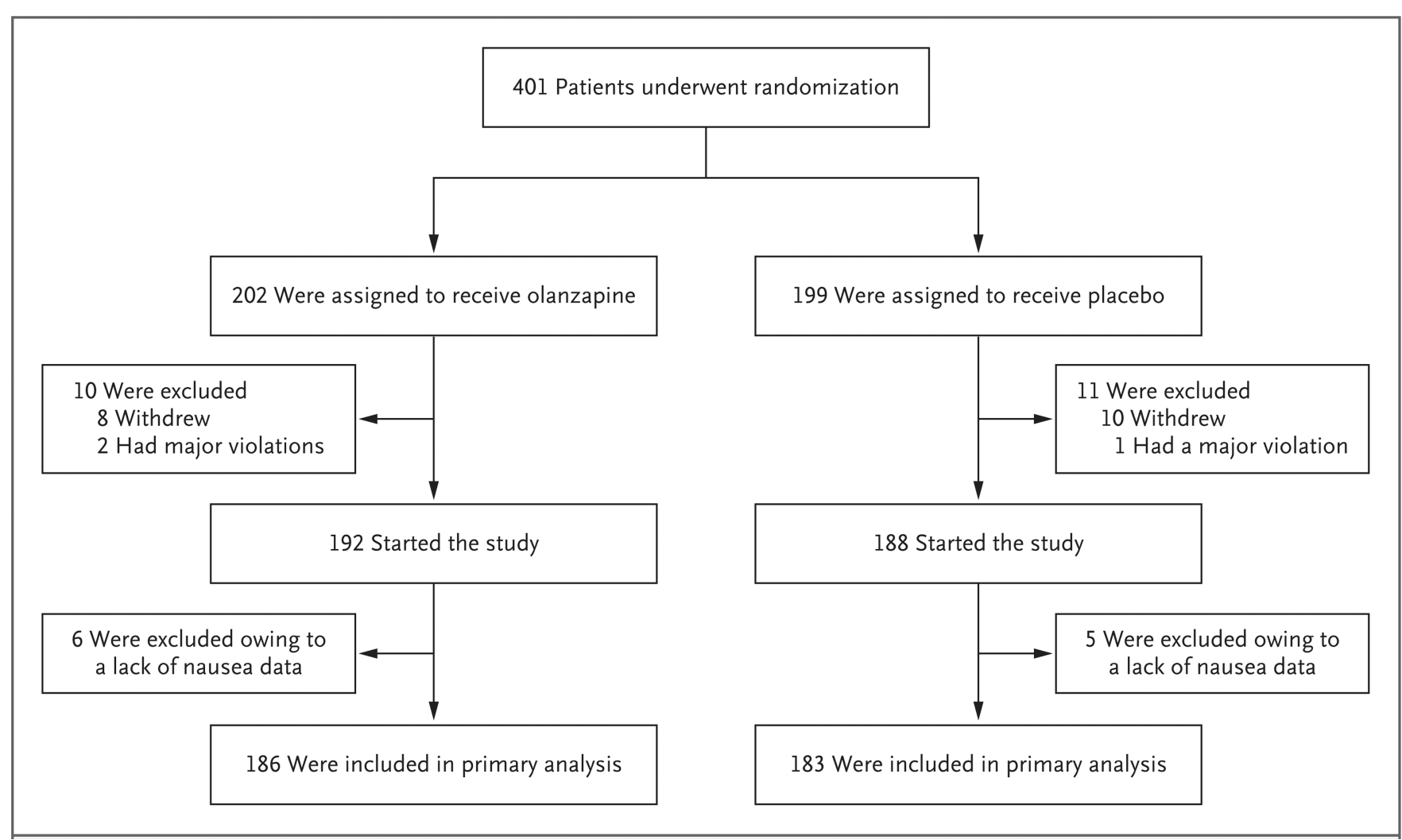

Figure 1. Patients Who Underwent Randomization, Started the Study, and Were Included in the Primary Analysis 


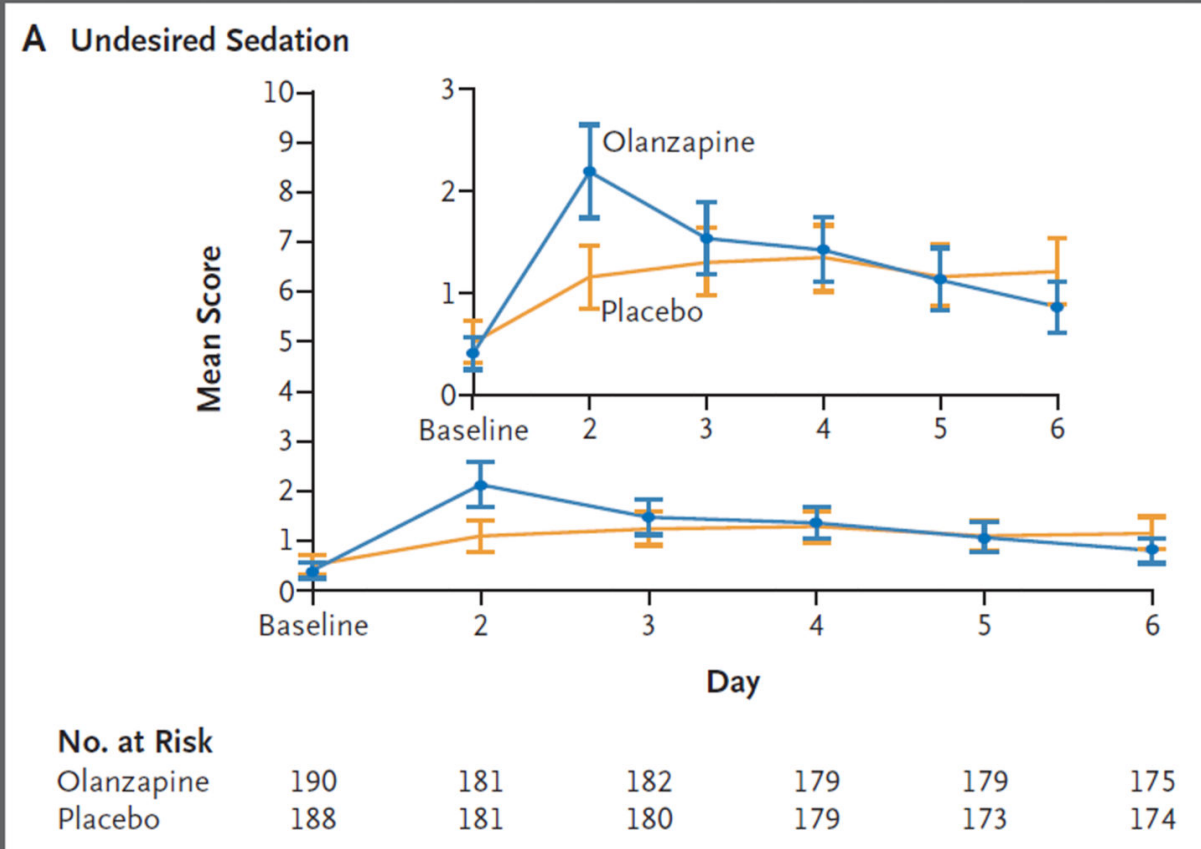

B Undesired Increase in Appetite

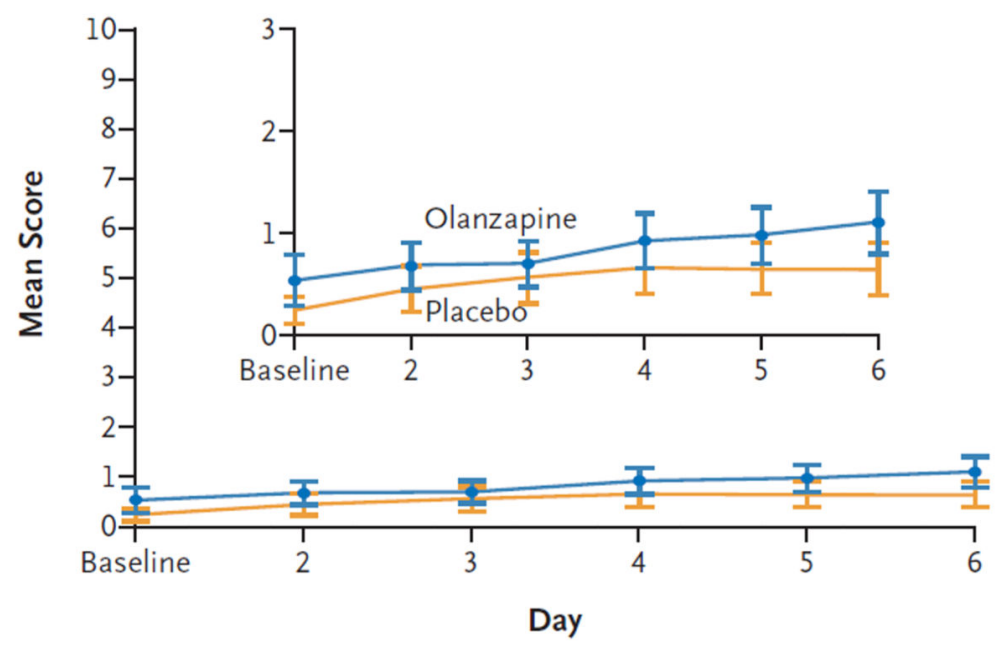

No. at Risk

Olanzapine

190

187

183

182

179

179

176

Placebo

181

180

179

174

174

Figure 2. Ratings of Undesired Sedation and Undesired Increase in Appetite in the Olanzapine and Placebo Groups

Patients were asked to record daily levels of undesired sedation and undesired increase in appetite using a visual-analogue scale ranging from 0 (none) to 10 ("as bad as it can be"). The mean scores for undesired sedation (Panel A) and undesired increase in appetite (Panel B) are shown for 5 days after chemotherapy. I bars indicate standard errors. 


\section{Table 1}

Baseline Demographic and Clinical Characteristics of the Study Patients. ${ }^{*}$

\begin{tabular}{|c|c|c|c|}
\hline Characteristic & $\begin{array}{c}\text { Olanzapine } \\
(\mathbf{N}=192)\end{array}$ & $\begin{array}{c}\text { Placebo } \\
(\mathbf{N}=188)\end{array}$ & $\begin{array}{c}\text { Total } \\
(\mathbf{N}=\mathbf{3 8 0})\end{array}$ \\
\hline \multicolumn{4}{|l|}{ Age $-\mathrm{yr}$} \\
\hline Median & 58.0 & 56.0 & 57.0 \\
\hline Range & $29.0-86.0$ & $28.0-89.0$ & $28.0-89.0$ \\
\hline \multicolumn{4}{|l|}{ Race or ethnic group - no. $(\%)^{\dagger}$} \\
\hline White & $172(89.6)$ & $171(91.0)$ & $343(90.3)$ \\
\hline Black & $9(4.7)$ & $9(4.8)$ & $18(4.7)$ \\
\hline Asian & $5(2.6)$ & $4(2.1)$ & $9(2.4)$ \\
\hline American Indian or Alaska Native & $3(1.6)$ & $1(0.5)$ & $4(1.1)$ \\
\hline Not assessed & $3(1.6)$ & $3(1.6)$ & $6(1.6)$ \\
\hline \multicolumn{4}{|l|}{ Sex - no. $(\%)$} \\
\hline Female & $139(72.4)$ & $136(72.3)$ & $275(72.4)$ \\
\hline Male & $53(27.6)$ & $52(27.7)$ & 105 (27.6) \\
\hline \multicolumn{4}{|l|}{ 5-HT ${ }_{3}$-receptor antagonist - no. $(\%)$} \\
\hline Palonosetron & $145(75.5)$ & $143(76.1)$ & $288(75.8)$ \\
\hline Ondansetron & $46(24.0)$ & $44(23.4)$ & $90(23.7)$ \\
\hline Granisetron & $1(0.5)$ & $1(0.5)$ & $2(0.5)$ \\
\hline \multicolumn{4}{|l|}{ Chemotherapy regimen - no. $(\%)$} \\
\hline Cisplatin-containing regimen & $71(37.0)$ & $65(34.6)$ & $136(35.8)$ \\
\hline Anthracycline and cyclophosphamide & $121(63.0)$ & $123(65.4)$ & $244(64.2)$ \\
\hline \multicolumn{4}{|l|}{ ECOG performance status - no. $(\%)^{*}$} \\
\hline 0 & $149(77.6)$ & $144(76.6)$ & $293(77.1)$ \\
\hline 1 & $40(20.8)$ & $41(21.8)$ & $81(21.3)$ \\
\hline 2 & $2(1.0)$ & $3(1.6)$ & $5(1.3)$ \\
\hline Not assessed & $1(0.5)$ & 0 & $1(0.3)$ \\
\hline \multicolumn{4}{|l|}{ Primary site of disease - no. $(\%)$} \\
\hline Breast & $120(62.5)$ & $122(64.9)$ & $242(63.7)$ \\
\hline Lung & $27(14.1)$ & $22(11.7)$ & $49(12.9)$ \\
\hline Other & $45(23.4)$ & $44(23.4)$ & $89(23.4)$ \\
\hline
\end{tabular}

"The Wilcoxon test was used to compare age between the two study groups, and Fisher's exact test was used for comparisons of the other characteristics. There were no significant between-group differences. Percentages may not total 100 because of rounding. 5-HT3 denotes 5hydroxytryptamine type 3 .

Race or ethnic group was self-reported.

${ }^{*}$ The European Cooperative Oncology Group (ECOG) performance status is measured on a 5-point scale, with 0 indicating no symptoms and higher numbers indicating increasing disability. 


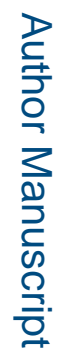

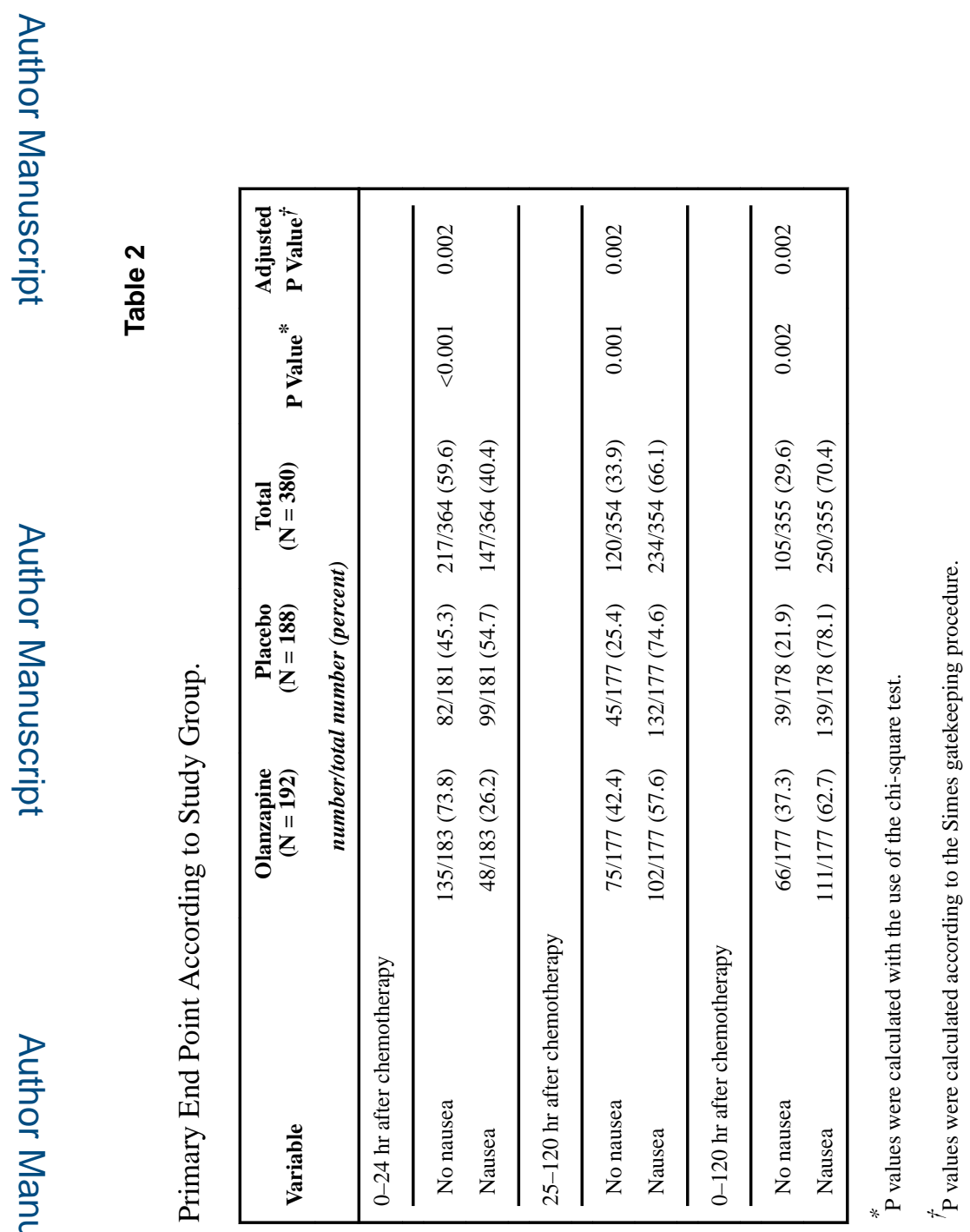

NEngl J Med. Author manuscript; available in PMC 2017 March 09. 


\section{롤 \\ ฏ.}

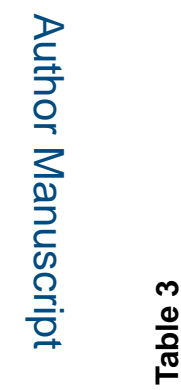

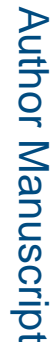

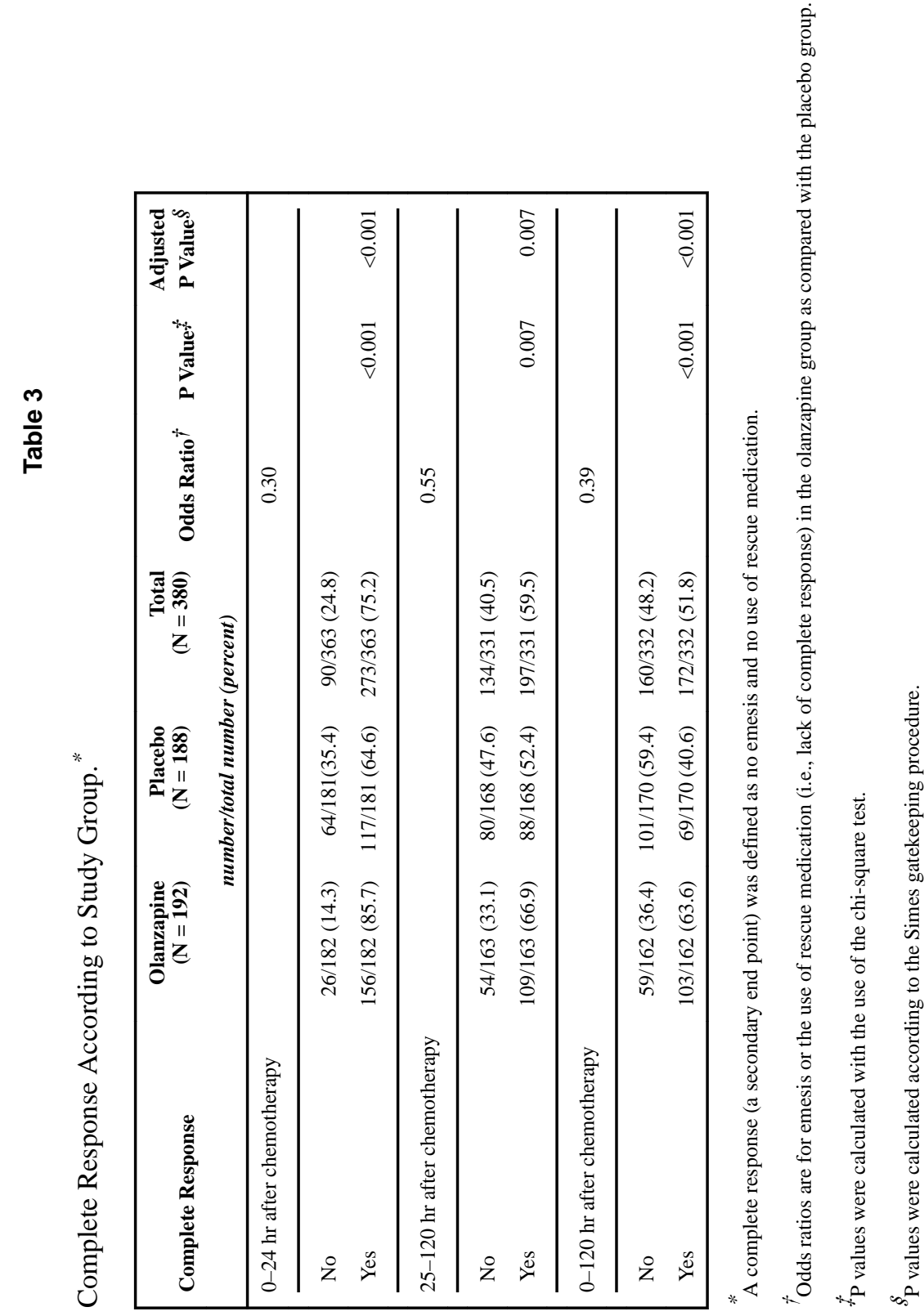

NEngl J Med. Author manuscript; available in PMC 2017 March 09. 